\title{
Outcome of Juvenile Idiopathic Arthritis Associated Uveitis in Two Disease Subtypes
}

\author{
Dragana LAZAREVIĆ, ${ }^{1}$ Ivana NIKOLIĆ, ${ }^{1}$ Marija RATKOVIĆ-JANKOVIĆ, ${ }^{1}$ Jelena VOJINOVIĆ ${ }^{1,2}$ \\ ${ }^{1}$ Department of Pediatric Rheumatology, Clinic of Pediatrics, Clinical Center Nǐ̌, Niš, Serbia \\ ${ }^{2}$ Medical Faculty, University of Niš, Niš, Serbia
}

\begin{abstract}
Objectives: This study aims to evaluate the efficacy of adalimumab as a first line biologic agent in specific subtypes of juvenile idiopathic arthritis (JIA) patients with associated uveitis.

Patients and methods: We retrospectively analyzed the data of 11 JIA patients ( 8 males, 3 females; mean age 14.5 years; range 9 to 18 years) with associated uveitis treated with biologic therapy. All patients were diagnosed as oligoarticular/extended oligoarticular or enthesitis-related JIA subtypes, treated with methotrexate, and had active or previous history of uveitis for which adalimumab was prescribed. We tested all patients for anti-nuclear antibody presence and human leukocyte antigen genotype. We assessed disease activity and therapy efficacy by American College of Rheumatology 50\%, 70\%, and 100\% improvement criteria. We evaluated uveitis activity by slit-lamp biomicroscopy and recorded adverse events.

Results: Of the JIA patients, three (27.27\%) had oligoarticular/extended oligoarticular JIA and eight (72.73\%) had enthesitis-related arthritis. Anti-nuclear antibody positivity was present in $27.27 \%$ (all females) while human leukocyte antigen-B51 was determined in $62.5 \%$ and human leukocyte antigen-B27 in $12.5 \%$ of patients. Mean uveitis duration before adalimumab introduction was 12.3 months. After two years of follow-up, there were no relapses of uveitis and visual acuity was stable while on adalimumab and methotrexate treatment. All patient were gradually tapered and discontinued treatment with topical steroids. Disease activity improved and seven patients (63.64\%) achieved American College of Rheumatology $100 \%$ response rate (attained remission), while four patients (36.36\%) achieved American College of Rheumatology $70 \%$ response rate.

Conclusion: Anti-nuclear antibody positivity with oligoarticular/extended oligoarticular and enthesitis-related arthritis JIA subtypes, which are known for their high risk to develop uveitis, may benefit from adalimumab as a first line anti-tumor necrosis factor agent.

Keywords: Adalimumab; juvenile idiopathic arthritis; uveitis.
\end{abstract}

Juvenile idiopathic arthritis (JIA) is the most common chronic rheumatic condition in children and comprises of a heterogeneous group of seven different diseases, ${ }^{1,2}$ Some JIA subtypes have increased susceptibility to develop inflammation of anterior or posterior chamber, known as uveitis. Antinuclear antibody (ANA)-positive females younger than seven years of age with oligoarticular/extended oligoarticular JIA are at greater risk to develop uveitis, as well as males older than six years of age with enthesitis-related arthritis (ERA) JIA. ${ }^{3}$ Uveitis is an important extraarticular manifestation of JIA present in 10 to $30 \%$ of patients. ${ }^{4}$ Chronic course, bilateral onset, and recurrent episodes are characteristics of childhood uveitis. Unfortunately, due to young age, uveitis may be asymptomatic or unrecognized. ${ }^{5}$ Usually, it is diagnosed within the first seven years after the onset of arthritis; however, structural damage is present in 30 to $50 \%$ of children when JIA diagnosis is established. ${ }^{6}$ Regular ophthalmological screening is recommended in these patients, particularly in certain JIA subtypes, to prevent visual loss., ${ }^{4,8}$ Without adequate treatment, chronic inflammation might lead to severe irreparable damage and structural changes with serious complications (posterior synechiae, band keratopathy, cataract, glaucoma, 
hypotonic, or macular edema) as well as blindness which may have additional impact on quality of life.

Regarding the impact of these facts on clinical decision and the importance of the choice of first biologic agents in everyday clinical practice, several years ago, we decided to implement specific practice to prescribe adalimumab preferably as the first anti-tumor necrosis factor (TNF) agent in all ANA positive oligoarticular/ extended oligoarticular and ERA JIA patients with associated uveitis at our center. Therefore, in this study, we aimed to evaluate the efficacy of adalimumab as a first biologic agent in specific subtypes of JIA patients with associated uveitis.

\section{PATIENTS AND METHODS}

We retrospectively analyzed the data of 11 JIA patients ( 8 males, 3 females; mean age 14.5 years; range 9 to 18 years) treated with biologic therapy from the registry of Clinic of Pediatrics, Clinical Center Niš between April 2010 and May 2012. All patients were diagnosed as ANA positive oligoarticular/ extended oligoarticular or ERA JIA subtypes, with active or previous history of uveitis for which adalimumab was prescribed if it failed to respond to standard treatment. All patients were regularly screened by an ophthalmologist for uveitis by slit-lamp biomicroscopy examination, according to the recommendations. ${ }^{4}$ Possible complications of chronic inflammation were assessed by intraocular pressure measurement and fundus examination. The number of flares and ocular complications were recorded. In case of acute new onset of uveitis or uveitis relapse flare, activity of inflammation in the eye was assessed weekly and monthly. In case of active ocular inflammation, patients were treated with topical treatment with steroids and cycloplegics in addition to disease-modifying antirheumatic drug therapy (methotrexate $10-20 \mathrm{mg} / \mathrm{m}^{2} /$ weekly). In case of unsatisfactory response, treatment with adalimumab was initiated (24 mg/m $\mathrm{m}^{2}$ in two weeks intervals). Study was approved by the Ethical Committee of the Medical Faculty, University of Nis, Serbia. and informed written consent was obtained from parents and/or patients. The study was conducted in accordance with the principles of the Declaration of Helsinki.

We used International League of Association for Rheumatology classification criteria for JIA diagnosis and subtype categorization. ${ }^{1}$ Registry had been systematically collecting demographic data of JIA patients such as sex, age, age at disease onset, disease duration, previous and concomitant therapy, disease activity, and treatment efficacy. We obtained blood samples every three months for routine laboratory monitoring of drug treatment safety. Additionally, we tested all patients for ANA presence and performed human leukocyte antigen (HLA) genotype test. We assessed disease activity and therapy efficacy by calculating the American College of Rheumatology Pediatric (ACR Pedi) score for 50\%, 70\%, and 100\% improvement criteria, using six core set variables: the physician global assessment of disease activity on a $10 \mathrm{~cm}$ visual analog scale (0-100), the parent/patient global assessment of overall well-being on a $10 \mathrm{~cm}$ visual analog scale (0-100), the Childhood Health Assessment Questionnaire (0-3), the numbers of joints with limited range of motion and active arthritis, and erythrocyte sedimentation rate. The definition of improvement states that there should be at least $50 \%$ of improvement (70\%, 100\%) from baseline in three out of six core set variables and no more than one core set criteria should worsen more than 30\%.9 Disease remission was considered according to the Wallace et al. criteria. $^{10}$

\section{Statistical analysis}

Statistical analysis was performed by SPSS software version 16.0 (SPSS Inc., Chicago, IL, USA). All results were reported as the mean \pm standard error, minimal and maximal values.

\section{RESULTS}

Detailed analysis of registry records produced data of 11 JIA patients who were followed-up for two years and diagnosed as active JIA associated uveitis treated with adalimumab. All patients were biologic treatment naive. Three patients (27.27\%) were diagnosed as oligoarticular/ extended oligoarticular while eight (72.73\%) were diagnosed as ERA JIA subtype. Detailed demographic and disease data are presented in 
Table 1. Demographic data of JIA patients with uveitis $(n=11)$

\begin{tabular}{lcccc}
\hline Characteristic & $\mathrm{n}$ & $\%$ & $\mathrm{Mean} \pm \mathrm{SD}$ & Min.-Max. \\
\hline Sex & 3 & 27.27 & & \\
$\quad$ Female & 5 & 72.73 & & \\
$\quad$ Male & 3 & 27.27 & & \\
Oligoarticular/extended oligoarticular JIA & 8 & 27.37 & $77.9 \pm 36.5$ & $27-156$ \\
Enthesitis related JIA & & & $111.8 \pm 54.1$ & $45-204$ \\
Age at JIA diagnosis, (months) & & & $48.3 \pm 39.1$ & $6-120$ \\
Age at uveitis diagnosis, (months) & & & $12.3 \pm 10.8$ & $3-36$ \\
Mean JIA duration, (months) & & & $47.2 \pm 39.2$ & $12-144$ \\
Mean uveitis duration, (months) & & & \\
Average duration of methotrexate therapy & & & & \\
Average dose of methotrexate & $10 \mathrm{mg} / \mathrm{m}^{2} /$ weekly & & & \\
SD: Standard deviation; Min.: Minimum; Max.: Maximum; JIA: Juvenile idiopathic arthritis. & & & \\
\hline
\end{tabular}

Table 1. Two patients had monocular visual loss, but treatment was introduced due to the acute uveitis in previously unaffected eye.

Of the 11 JIA patients, 27.3\% (all females) were ANA positive. Genetic analysis showed HLAB51 positivity in $62.5 \%$ and HLAB27 positivity in $12.5 \%$ in male patients while all female patients were negative.

During the two-year follow up period of adalimumab treatment, there were no relapses of uveitis and visual acuity was stable. All patients were gradually tapered and treatment was continued with topical steroids after approximately four months of adalimumab treatment. At the end of the observational period, only two patients (18.18\%) had monocular visual loss which was present before the commencement of adalimumab therapy. Disease activity, measured by ACR response rates, improved in seven patients (63.64\%) achieving ACR 100\% response rate (attained remission) and four patients (36.36\%) achieving ACR $70 \%$ response rate (Figure 1). We recorded no serious adverse events except for pain at the injection site.

\section{DISCUSSION}

Juvenile idiopathic arthritis associated uveitis is one of the most severe extra-articular manifestations in JIA. It is estimated that 20 to $30 \%$ of JIA patients are at risk to develop

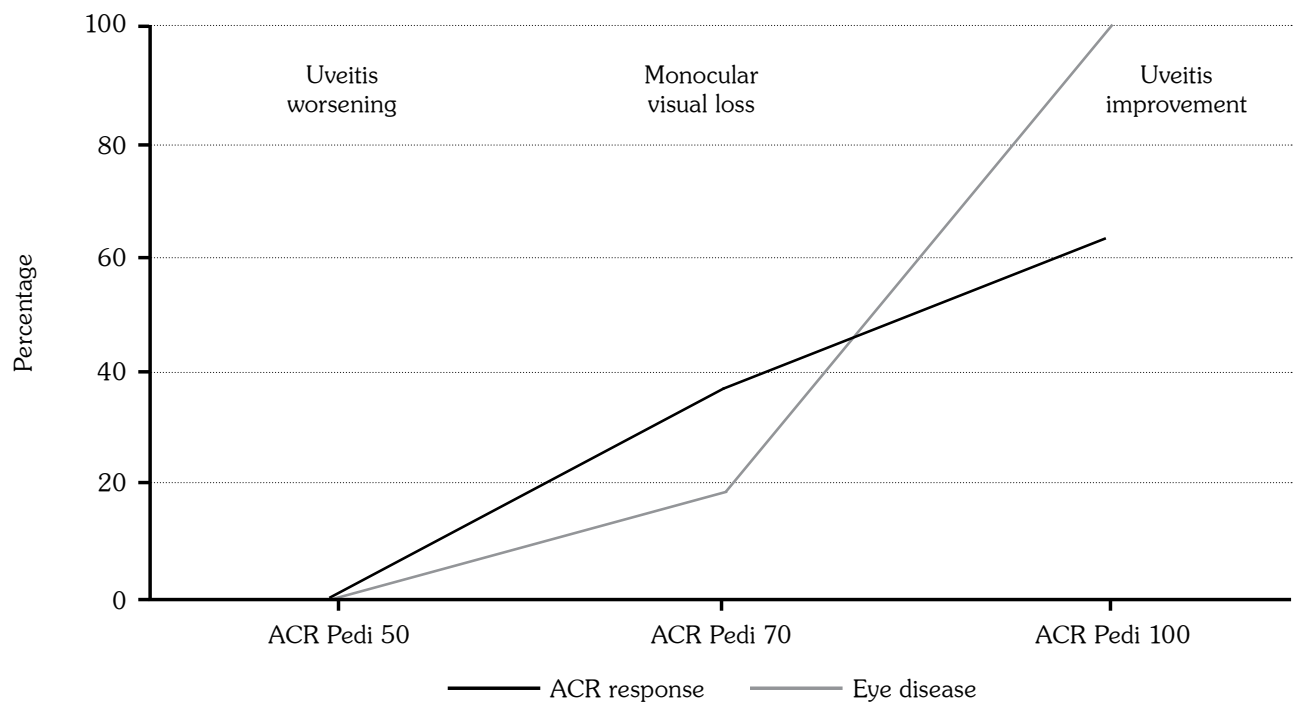

Figure 1. Efficacy of adalimumab on juvenile idiopathic arthritis and juvenile idiopathic arthritis associated uveitis. JIA: Juvenile idiopathic arthritis; ACR Pedi: American College of Rheumatology Pediatric Criteria. 
uveitis with consequent loss of visual acuity or blindness. ${ }^{5,11-13}$ Initial treatment with topical steroids, cycloplegics, and disease modifying drugs are often insufficient to control disease activity and may lead to serious structural damage. ${ }^{11,14,15}$ Therefore, certain research groups have developed a set of new treatment and follow-up recommendations. ${ }^{4,16}$

Improvements in anti-TNF-alpha drugs have changed long-term outcomes in patients who did not respond to second line disease-modifying antirheumatic drugs. ${ }^{3,16-20}$ Different biologics targeting the pivotal cytokine TNF-alpha in pathogenesis of these chronic diseases were used with different efficacy in JIA associated uveitis. Infliximab and etanercept were the first biologics available for usage, with beneficial effects on the suppression of the disease activity in eye and joints, ${ }^{21,22}$ but etanercept had shown less effectiveness in controlling ocular inflammation than infliximab. ${ }^{20,23}$ Some published data even indicated that etanercept might cause relapses of uveitis, or induce its onset. ${ }^{24}$ Adalimumab has been shown to be effective not only in treating JIA, but also in refractory cases of intraocular inflammation. ${ }^{3,18,25-28}$ In their prospective comparative study, Simonini et al. ${ }^{29}$ have demonstrated superior efficacy of adalimumab compared to infliximab in the treatment of refractory chronic uveitis in JIA patients. Similar findings were reported in an analysis of the Italian National Registry ${ }^{30}$ Consequently, several years ago, researchers of our center decided to implement specific clinical practice to prescribe preferably adalimumab as the first anti-TNF agent in all ANA positive oligoarticular/extended oligoarticular and ERA JIA patients with JIA associated uveitis. ${ }^{12}$ This is in accordance with a recent study of Tappeiner et al. ${ }^{31}$ who reported lack of efficacy of adalimumab on ocular disease in JIA patients with severe uveitis and frequent flares of uveitis where adalimumab was not the first anti-TNFalpha treatment choice. ${ }^{31}$

Recent data published from German BIKER biological registry demonstrated that the risk of uveitis flare was similar in patients with positive history of uveitis treated with etanercept or adalimumab. ${ }^{32}$ Same study has reported no new uveitis events upon treatment with adalimumab. It is interesting to note that
German pediatric rheumatologists, similar to us, preferably prescribed adalimumab to JIA patients who had a history of previous uveitis and have been diagnosed as oligoarticular/extended oligoarticular JIA; however, this was not the case for ERA patients.

Presence of HLAB27 and HLAB51 haplotypes as genetic susceptibility factors associated with development of JIA uveitis has been confirmed in our small cohort. ${ }^{3}$ Additionally, ANA positivity was found in all female JIA patients with associated uveitis similarly to the well-known data. ${ }^{11,12,16,33,34}$ After two years of follow-up, our results have shown good clinical response of the eye inflammatory process and the joint disease activity while on treatment with adalimumab in combination with methotrexate. Different authors have reported the same findings. ${ }^{35,36}$ The ongoing SYCAMORE trial has been investigating the clinical effectiveness and safety of dual adalimumab and methotrexate treatment of JIA associated uveitis. ${ }^{6}$ Our results have shown no new flares or worsening of visual acuity and only two patients had monocular visual loss, which was already present before starting adalimumab treatment. Similar findings have been reported by several reports. ${ }^{28,29,35}$ We should highlight that our patients were previously biologically naive, which might explain the high efficacy obtained. This is in accordance with the data of Simonini et al., ${ }^{37}$ who also demonstrated better efficacy of adalimumab when used as a first anti-TNF-alpha treatment in chronic childhood uveitis. Steroid sparing effects, found in our patient cohort, enabled reduction and complete ending of steroids after commencing adalimumab and methotrexate, which is in concordance with published data. ${ }^{38,39}$ The fact that no serious adverse events were recorded during the followup period indicates that safety profile of these therapeutic options is high. Patients only reported pain at the injection site.

A limitation of our study is the small sample size, disallowing to make a direct comparison between.

In conclusion, our study indicates that ANA positive oligoarticular/extended oligoarticular and ERA JIA subtypes, known for their high risk to develop uveitis, may benefit from adalimumab as a first-line anti-TNF agent. 


\section{Declaration of conflicting interests}

The authors declared no conflicts of interest with respect to the authorship and/or publication of this article.

\section{Funding}

The authors acknowledge financial support from the Ministry of Science and Technological Development of Serbia (Project 41018).

\section{REFERENCES}

1. Petty RE, Southwood TR, Manners P, Baum J, Glass $\mathrm{DN}$, Goldenberg $\mathrm{J}$, et al. International League of Associations for Rheumatology classification of juvenile idiopathic arthritis: second revision, Edmonton, 2001. J Rheumatol 2004;31:390-2.

2. Beukelman T, Patkar NM, Saag KG, Tolleson-Rinehart S, Cron RQ, DeWitt EM, et al. 2011 American College of Rheumatology recommendations for the treatment of juvenile idiopathic arthritis: initiation and safety monitoring of therapeutic agents for the treatment of arthritis and systemic features. Arthritis Care Res (Hoboken) 2011;63:465-82.

3. Kotaniemi K, Säilä H, Kautiainen H. Long-term efficacy of adalimumab in the treatment of uveitis associated with juvenile idiopathic arthritis. Clin Ophthalmol 2011;5:1425-9.

4. Bou R, Adán A, Borrás F, Bravo B, Calvo I, De Inocencio $\mathrm{J}$, et al. Clinical management algorithm of uveitis associated with juvenile idiopathic arthritis: interdisciplinary panel consensus. Rheumatol Int 2015;35:777-85.

5. Bou R, Iglesias E, Antón J. Treatment of uveitis associated with juvenile idiopathic arthritis. Curr Rheumatol Rep 2014;16:437.

6. Ramanan AV, Dick AD, Benton D, CompeyrotLacassagne S, Dawoud D, Hardwick B, et al. A randomised controlled trial of the clinical effectiveness, safety and cost-effectiveness of adalimumab in combination with methotrexate for the treatment of juvenile idiopathic arthritis associated uveitis (SYCAMORE Trial). Trials 2014;15:14.

7. Heiligenhaus A, Niewerth M, Ganser G, Heinz C, Minden K. Prevalence and complications of uveitis in juvenile idiopathic arthritis in a population-based nation-wide study in Germany: suggested modification of the current screening guidelines. Rheumatology (Oxford) 2007;46:1015-9.

8. Cassidy J, Kivlin J, Lindsley C, Nocton J. Ophthalmologic examinations in children with juvenile rheumatoid arthritis. Pediatrics 2006;117:1843-5.

9. Giannini EH, Ruperto N, Ravelli A, Lovell DJ, Felson DT, Martini A. Preliminary definition of improvement in juvenile arthritis. Arthritis Rheum 1997;40:1202-9.

10. Wallace CA, Ruperto N, Giannini E. Preliminary criteria for clinical remission for select categories of juvenile idiopathic arthritis. J Rheumatol 2004;31:2290-4.

11. Gregory AC, Kempen JH, Daniel E, Kaçmaz RO, Foster CS, Jabs DA, et al. Risk factors for loss of visual acuity among patients with uveitis associated with juvenile idiopathic arthritis: the Systemic Immunosuppressive Therapy for Eye Diseases Study. Ophthalmology 2013;120:186-92.

12. Saurenmann RK, Levin AV, Feldman BM, Rose JB, Laxer RM, Schneider R, et al. Prevalence, risk factors, and outcome of uveitis in juvenile idiopathic arthritis: a long-term followup study. Arthritis Rheum 2007;56:647-57.

13. Rodríguez-García A. The importance of an ophthalmologic examination in patients with juvenile idiopathic arthritis. Reumatol Clin 2015;11:133-8.

14. Rosenberg KD, Feuer WJ, Davis JL. Ocular complications of pediatric uveitis. Ophthalmology 2004;111:2299-306.

15. Foeldvari I. Ocular Involvement in Juvenile Idiopathic Arthritis: Classification and Treatment. Clin Rev Allergy Immunol 2015;49:271-7.

16. Heiligenhaus A, Michels H, Schumacher C, Kopp I, Neudorf U, Niehues T, et al. Evidence-based, interdisciplinary guidelines for anti-inflammatory treatment of uveitis associated with juvenile idiopathic arthritis. Rheumatol Int 2012;32:1121-33.

17. Cordero-Coma M, Sobrin L. Anti-tumor necrosis factor- $\alpha$ therapy in uveitis. Surv Ophthalmol 2015;60:575-89.

18. Tynjälä $\mathrm{P}$, Kotaniemi K, Lindahl $\mathrm{P}$, Latva K, Aalto $\mathrm{K}$, Honkanen $\mathrm{V}$, et al. Adalimumab in juvenile idiopathic arthritis-associated chronic anterior uveitis. Rheumatology (Oxford) 2008;47:339-44.

19. Foeldvari I, Nielsen S, Kümmerle-Deschner J, Espada G, Horneff G, Bica B, et al. Tumor necrosis factoralpha blocker in treatment of juvenile idiopathic arthritis-associated uveitis refractory to second-line agents: results of a multinational survey. J Rheumatol 2007;34:1146-50.

20. Saurenmann RK, Levin AV, Rose JB, Parker S, Rabinovitch T, Tyrrell PN, et al. Tumour necrosis factor alpha inhibitors in the treatment of childhood uveitis. Rheumatology (Oxford) 2006;45:982-9.

21. Reiff A, Takei S, Sadeghi S, Stout A, Shaham B, Bernstein $B$, et al. Etanercept therapy in children with treatment-resistant uveitis. Arthritis Rheum 2001;44:1411-5.

22. Schmeling H, Horneff G. Etanercept and uveitis in patients with juvenile idiopathic arthritis. Rheumatology (Oxford) 2005;44:1008-11.

23. Tynjälä $\mathrm{P}$, Lindahl $\mathrm{P}$, Honkanen $\mathrm{V}$, Lahdenne $\mathrm{P}$, Kotaniemi K. Infliximab and etanercept in the treatment of chronic uveitis associated with refractory juvenile idiopathic arthritis. Ann Rheum Dis 2007;66:548-50.

24. Neri P, Zucchi M, Allegri P, Lettieri M, Mariotti $C$, Giovannini A. Adalimumab (Humira ${ }^{\mathrm{TM}}$ ): a promising 
monoclonal anti-tumor necrosis factor alpha in ophthalmology. Int Ophthalmol 2011;31:165-73.

25. Vazquez-Cobian LB, Flynn T, Lehman TJ. Adalimumab therapy for childhood uveitis. J Pediatr 2006;149:572-5.

26. Ruperto N, Lovell DJ, Quartier P, Paz E, RubioPérez N, Silva CA, et al. Abatacept in children with juvenile idiopathic arthritis: a randomised, doubleblind, placebo-controlled withdrawal trial. Lancet 2008;372:383-91.

27. Sen ES, Sharma S, Hinchcliffe A, Dick AD, Ramanan AV. Use of adalimumab in refractory non-infectious childhood chronic uveitis: efficacy in ocular disease-a case cohort interventional study. Rheumatology (Oxford) 2012;51:2199-203.

28. La Torre F, Cattalini M, Teruzzi B, Meini A, Moramarco $F$, Iannone F. Efficacy of adalimumab in young children with juvenile idiopathic arthritis and chronic uveitis: a case series. BMC Res Notes 2014;7:316.

29. Simonini G, Taddio A, Cattalini M, Caputo R, De Libero $\mathrm{C}$, Naviglio $\mathrm{S}$, et al. Prevention of flare recurrences in childhood-refractory chronic uveitis: an open-label comparative study of adalimumab versus infliximab. Arthritis Care Res (Hoboken) 2011;63:612-8.

30. Zannin ME, Birolo C, Gerloni VM, Miserocchi E, Pontikaki I, Paroli MP, et al. Safety and efficacy of infliximab and adalimumab for refractory uveitis in juvenile idiopathic arthritis: 1-year followup data from the Italian Registry. $\mathrm{J}$ Rheumatol 2013;40:74-9.

31. Tappeiner C, Miserocchi E, Bodaghi B, Kotaniemi $\mathrm{K}$, Mackensen F, Gerloni V, et al. Abatacept in the treatment of severe, longstanding, and refractory uveitis associated with juvenile idiopathic arthritis. J Rheumatol 2015;42:706-11.
32. Foeldvari I, Becker I, Horneff G. Uveitis Events During Adalimumab, Etanercept, and Methotrexate Therapy in Juvenile Idiopathic Arthritis: Data From the Biologics in Pediatric Rheumatology Registry. Arthritis Care Res (Hoboken) 2015;67:1529-35.

33. Saurenmann RK, Levin AV, Feldman BM, Laxer RM, Schneider R, Silverman ED. Risk factors for development of uveitis differ between girls and boys with juvenile idiopathic arthritis. Arthritis Rheum 2010;62:1824-8.

34. Heiligenhaus A, Minden K, Föll D, Pleyer U. Uveitis in juvenile idiopathic arthritis. Dtsch Arztebl Int 2015;112:92-100.

35. Magli A, Forte R, Navarro P, Russo G, Orlando F, Latanza L, et al. Adalimumab for juvenile idiopathic arthritis-associated uveitis. Graefes Arch Clin Exp Ophthalmol 2013;251:1601-6.

36. Lovell DJ, Ruperto N, Goodman S, Reiff A, Jung L, Jarosova K, et al. Adalimumab with or without methotrexate in juvenile rheumatoid arthritis. $\mathrm{N}$ Engl J Med 2008;359:810-20.

37. Simonini G, Taddio A, Cattalini M, Caputo R, de Libero C, Parentin F, et al. Superior efficacy of Adalimumab in treating childhood refractory chronic uveitis when used as first biologic modifier drug: Adalimumab as starting anti-TNF- $\alpha$ therapy in childhood chronic uveitis. Pediatr Rheumatol Online J 2013;11:16.

38. García-De-Vicuña C, Díaz-Llopis M, Salom D, Bou R, Díaz-Cascajosa J, Cordero-Coma M, et al. Usefulness of adalimumab in the treatment of refractory uveitis associated with juvenile idiopathic arthritis. Mediators Inflamm 2013;2013:560632.

39. Lerman MA, Burnham JM, Chang PY, Daniel E, Foster $\mathrm{CS}$, Hennessy S, et al. Response of pediatric uveitis to tumor necrosis factor- $\alpha$ inhibitors. J Rheumatol 2013;40:1394-403. 